Short Note

\title{
A Paradox of Decreasing Entropy in Multiscale Monte Carlo Grain Growth Simulations
}

\author{
Michael Nosonovsky * and Sven K. Esche
}

Department of Mechanical Engineering, Stevens Institute of Technology, Hoboken, NJ 07030, USA

* Author to whom correspondence should be addressed; E-mail: Nosonovsky@yahoo.com

Received: 20 March 2008; in revised form: 9 June 2008 / Accepted: 14 June 2008 /

Published: 16 June 2008

\begin{abstract}
Grain growth in metals is driven by random thermal fluctuations and increases the orderliness of the system. This random process is usually simulated by the Monte Carlo (MC) method and Cellular Automata (CA). The increasing orderliness results in an entropy decrease, thus leading to a paradoxical apparent violation of the second law of thermodynamics. In this paper, it is shown that treating the system as a multiscale system resolves this paradox. $\mathrm{MC} / \mathrm{CA}$ simulations usually take into consideration only the mesoscale entropy. Therefore, the information entropy of the system decreases, leading to an apparent paradox. However, in the physical system, the entropy is produced at the nanoscale while it is consumed at the mesoscale, so that the net entropy is growing.
\end{abstract}

Keywords: grain growth, Monte Carlo simulation, Cellular Automata, multiscale modeling.

The entropy as a measure of irreversibility and dissipation in a thermodynamic system was introduced by R. Clausius in the 1850s. According to the second law of thermodynamics, the entropy of a closed system cannot decrease. In 1877, L. Boltzmann suggested a definition of the entropy using the statistical thermodynamics approach and the concept of microstates $\Omega$ corresponding to a given macrostate,

$$
S=k_{B} \ln \Omega
$$

where $k_{B}$ is Boltzmann's constant and $\Omega$ is the number of microstates. 
About 70 years later, the concept of information entropy was introduced by C. Channon, who defined it as

$$
H=K \ln (1 / p)
$$

where $K$ is a constant and $p$ is the probability associated with the information. The relationship between the two types of the entropy remains a matter of debate [1]. The extensive study of selforganizing systems since 1960 attracted attention to situations when the entropy decreases. However, these self-organizing systems are not closed and operate far from the equilibrium, with the entropy being driven away from the system.

Figure 1. MC simulation of grain growth (a) experimental observation of grains in aluminum at different time intervals (b) simulation lattice (c) simulation results for grain growth [2].

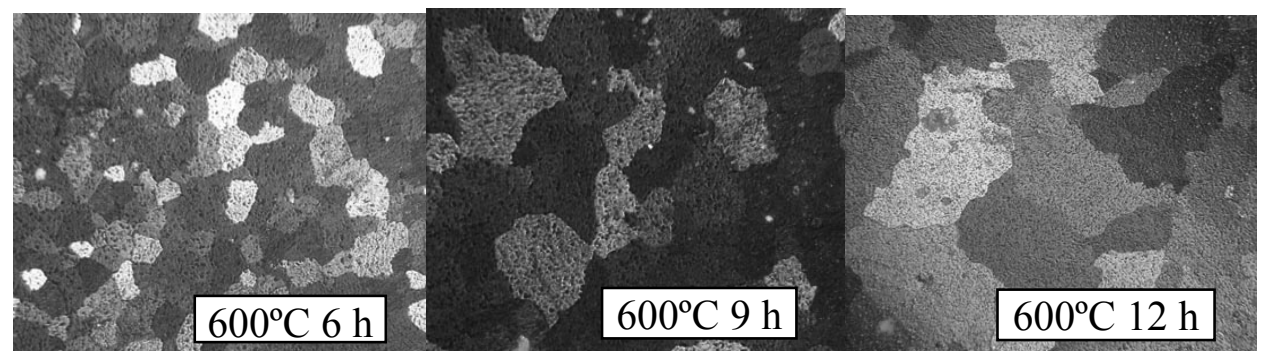

(a)

$$
\begin{aligned}
& \begin{array}{llllllll}
2 & 2 & 2 & 3 & 4 & 4 & 4 & 4
\end{array} \\
& \begin{array}{llll|llll}
2 & 2 & 3 & 3 & 4 & 4 & 4 & 4 \\
\hline 3 & 3 & 3 & 6 & 6 & 6 & 5 & 5
\end{array} \\
& \begin{array}{llllllll}
3 & 3 & 6 & 6 & 6 & 5 & 5 & 5
\end{array} \\
& \begin{array}{llllllll}
3 & 3 & 6 & 6 & 6 & 5 & 5 & 5
\end{array}
\end{aligned}
$$

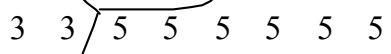

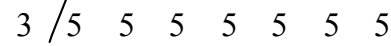

(b)
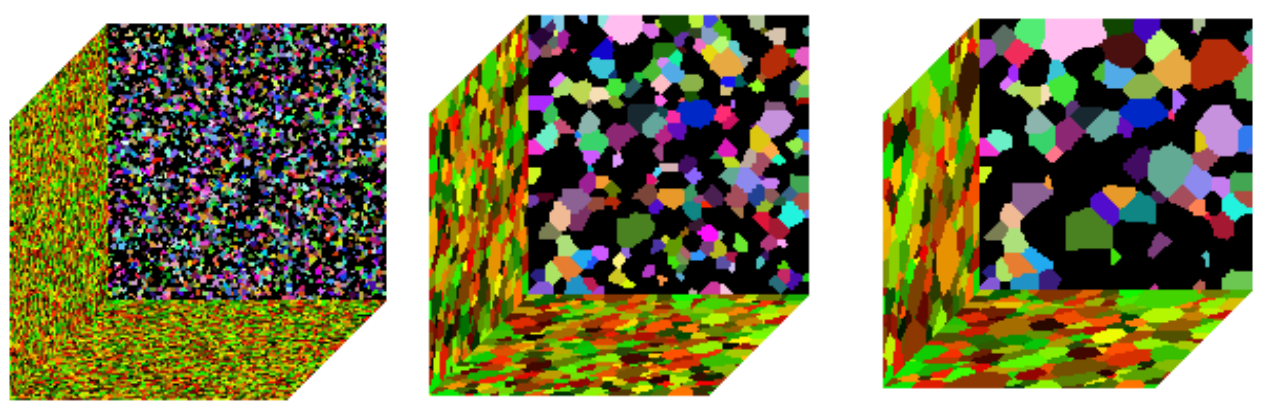

(c)

An interesting example of a self-organizing process is the thermally activated grain growth in metals (Fig. 1, [2]). It is well known that metal crystals form grains, characterized by different orientations of the lattice [3]. The typical grain size is in the range between microns and millimeters. There is an additional energy associated with the grain boundaries due to the misorientation of the neighboring grains. Therefore, it is energetically profitable for larger grains to grow and to absorb smaller grains, thus reducing the total number of grains and the total boundary area. The ideal state of 
a perfect crystal with only one grain corresponds to the minimum energy. The grain growth is a thermally activated diffusion-like process with the Arrhenius type of kinetics. For normal isotropic grain growth, theoretical considerations predict a parabolic dependence of the average grain radius $r$ on time

$$
r^{2}-r_{0}^{2}=K_{0} \exp \left(-\frac{Q}{R T}\right) t
$$

where $Q$ is the activation enthalpy, $R$ is the universal gas constant, $K_{0}$ is a proportionality constant and $r_{0}$ is the initial grain size [4].

Modeling the grain growth is a challenging task. Since it is a random process, deterministic modeling is difficult. Monte Carlo (MC) simulations and Cellular Automata (CA) approaches are often used [5-6]. CA algorithms describe the spatial and temporal evolution of a complex system by applying deterministic or probabilistic local rules to the cells of a regular lattice. They are based on finite difference formulations of local interaction laws. The MC method uses randomly generated orientation numbers associated with a regular lattice. The latter is initialized by randomly assigning to each lattice point an integer number representing its orientation. Reorientations are randomly and sequentially attempted for all lattice sites. If a new orientation is characterized by lower energy, then it is accepted [5-11].

The grains of a metal represent a typical example of a partially ordered system that occupies an intermediate position between the complete disorder (a random collection of atoms) and complete order (a perfect single crystal, which is close to the state of complete order, although it may have thermal equilibrium defects introducing disorder). The grain growth is also a self-organizing process system, which naturally evolves from a disordered to an ordered state, while being driven by random thermal fluctuations. Consider an array of $N$ cells, representing lattice cells in the simulation method, with each cell being in one of $M$ microstates. The total number of microstates of this system would then be:

$$
\Omega=M^{N}
$$

When the system reaches its final state with all cells having the same orientation, the total number of microstates becomes:

$$
\Omega=M
$$

Using the statistical mechanics definition of the entropy, one can find the initial entropy $S_{0}$ and the final entropy $S_{f}$ of the system as:

$$
\begin{aligned}
& S_{0}=\ln (M N)=N \ln M=N S_{f} \\
& S_{f}=\ln M
\end{aligned}
$$

Based on Eqs. 6 and 7, the entropy decreases between the initial and the final states by an amount of

$$
\Delta S=(N-1) \ln M
$$


This result constitutes an apparent paradox, because it seems to contradict the second law of thermodynamics, which states that the entropy of a closed system does not decrease. Another formulation of the paradox is that the randomness of thermal fluctuations leads to an increased orderliness of the system.

In order to solve this paradox, one has to take into account that the system under consideration can be studied at different scale levels, and also that it is not a closed system. At the macroscale, the continuum system is characterized by certain bulk mechanical properties (e.g., yield strength, elastic modulus) that depend upon the average grain size. At the macroscale, no change of entropy is expected, i.e. $\delta S_{\text {macro }}=0$. The mesoscale (or microscale) is governed by grains that tend to grow. Therefore, the mesoscale entropy is essentially the configuration entropy, and with increasing grain size it decreases due to the increased orderliness of the system, i.e. $\delta S_{\text {meso }}<0$. At the molecular scale (or nanoscale), energy barriers for grain growth and random thermal fluctuations exist. Every time when a lattice site is reoriented, a certain amount of energy is dissipated because the energy barrier must be overcome. However, if the system's temperature is maintained constant, then the heat is removed from the system due to its contact with the surroundings, and thus the system is not closed. This results in an increase in the system's temperature and entropy, i.e. $\delta S_{\text {nano }}>0$. In other words, the random fluctuations at the nanoscale lead to the orderliness increase at the mesoscale, which is compensated by the entropy increase at the nanoscale. The net entropy of the system can therefore be written as

$$
S_{\text {net }}=S_{\text {macro }}+S_{\text {meso }}+S_{\text {nano }}
$$

Since $\left|\delta S_{\text {nano }}\right|>\left|\delta S_{\text {meso }}\right|$, the net entropy decreases and the second law of thermodynamics is satisfied (Table 1).

Table 1. Hierarchy levels in the physical system and simulation.

\begin{tabular}{|c|c|c|c|c|c|c|}
\hline & \multicolumn{3}{|c|}{ Physical system } & \multicolumn{3}{c|}{ Simulation } \\
\cline { 2 - 7 } & Objects & $\begin{array}{c}\text { Driving } \\
\text { force }\end{array}$ & Entropy & Objects & Driving force & Entropy \\
\hline Macroscale & $\begin{array}{c}\text { Continuum } \\
\text { material }\end{array}$ & - & $\delta S_{\text {macro }}=0$ & - & - & - \\
\hline Mesoscale & Grains & $\begin{array}{c}\text { Decreasing } \\
\text { boundary } \\
\text { energy }\end{array}$ & $\delta S_{\text {meso }}<0$ & Cells & $\begin{array}{c}\text { Decreasing } \\
\text { energy } \\
\text { functional }\end{array}$ & $\delta S<0$ \\
\hline Nanoscale & Atoms & $\begin{array}{c}\text { Thermal } \\
\text { fluctuations }\end{array}$ & $\delta S_{\text {nano }}>0$ & - & - & - \\
\hline
\end{tabular}

Therefore, we deal with the two different systems: the physical system that is characterized by the thermodynamic entropy and the simulation system that models the physical system and is 
characterized by the information entropy. Concerning the information entropy of the system, several interesting observations can be made with regard to the self-organizing properties of this system. The numerical $\mathrm{CA} / \mathrm{MC}$ models consider only the mesoscale structure of the system, and thus only the mesoscale (configurational) entropy $S_{\text {meso }}$ can be determined from the CA/MC models. This is the reason for the apparent entropy decrease. The physical system is not insulated. The heat and entropy are removed from the system, and thus, it is not a closed system. However, the numerical CA/MC models do not take into account that aspect, because they are concentrated on the mesoscale description of the physical system. As a result, the second law of thermodynamics is apparently violated in the simulation system in a paradoxical manner. The second law is valid for closed physical systems. However, there is no reason why it cannot be violated for CA/MC models during computer simulation. One should keep in mind that computers require energy dissipation (i.e. computer processors require permanent cooling), and therefore, the decrease of entropy in the information system is compensated by an increase of the thermodynamic entropy.

The conclusion is that there is a correspondence between the physical system (a metal crystal) and the information system, used for the simulation (e.g. CA). However, the physical system is a multiscale system, with the entropy produced at the nanoscale and consumed at the mesoscale, so that the net sum of the entropy grows. The information system simulates only the mesoscale level, thus leading to the apparent reduction of the entropy. Consequently, the second law of thermodynamics is not observed within the computer simulation network. It is noted that such a common dissipative process as dry friction also has a hierarchical (multiscale) organization [12-13], and therefore the hierarchical approach to the entropy is important for a broad class of physical systems.

\section{Acknowledgements}

The development of the MC method was funded by the National Science Foundation through Award No. 0300273. This support is gratefully acknowledged. Furthermore, the authors would like to thank Dr. Qiang Yu for the programming of the MC method and Mr. Yujie Wu for the experimental measurements of the grain growth in aluminum samples.

\section{References and Notes}

1. Lin, S.-K. Diversity and Entropy. Entropy 1999, 1, 1-3.

2. Nosonovsky, M.; Zhang, X.; Esche, S. K. Scaling of Monte Carlo simulations of grain growth in metals. Comput. Mater. Sci. 2008, (submitted).

3. Humphreys, F. J.; Hatherly, M. Recrystallization and Related Annealing Phenomena; Pergamon: Oxford, 1995.

4. Yu, Q.; Nosonovsky, M.; Esche, S. K. On the accuracy of Monte Carlo Potts models for grain growth. J. Comp. Meth. Sci. Eng. 2008, (submitted).

5. Raabe, D. Scaling Monte Carlo kinetics of the Potts model using rate theory. Acta Materialia, 2000, 48, 1617-1628.

6. Raabe, D. Cellular Automata in materials science with particular reference to recrystallization simulation. Annual Review of Materials Research 2002, 32, 53-76. 
7. Saito, Y.; Enomoto, M. Monte Carlo simulation of grain growth. ISIJ International 1992, 32, 267274.

8. Yang, Z.; Sista, S.; Elmer, J. W.; Debroy, T. Three-dimensional Monte Carlo simulation of grain growth during GTA welding of titanium. Acta Mater. 2000, 48, 4813-4825.

9. Kobayashi, M.; Takayama, Y.; Kao, H. Prediction of microstructural evolution during grain growth of a pure aluminum by means of Monte Carlo simulation. Materials Transactions 2001, $42,2307-2315$.

10. Ding, R.; Guo, Z. X., Coupled quantitative simulation of microstructural evolution and plastic flow during dynamic recrystallization. Acta Materialia 2001, 49, 3163-3175.

11. Moldovan, D.; Wolf, D.; Phillpot, S. R; Haslam, A. J. Mesoscopic simulation of two-dimensional grain growth with anisotropic grain-boundary properties. Phil. Mag. A 2002, 82, 1271-1297.

12. Nosonovsky, M.; Bhushan, B. Multiscale friction mechanisms and hierarchical surfaces in nanoand bio-tribology. Mater. Sci. Eng. R 2007, 58, 162-193.

13. Nosonovsky, M.; Bhushan, B. Multiscale Dissipative Mechanisms and Hierarchical Surfaces: Friction, Superhydrophobicity, and Biomimetics; Springer Verlag: Heidelberg, 2008; Series on NanoScience and Technology, (in press).

(C) 2008 by the authors; licensee Molecular Diversity Preservation International, Basel, Switzerland. This article is an open-access article distributed under the terms and conditions of the Creative Commons Attribution license (http://creativecommons.org/licenses/by/3.0/). 